\title{
Analytic results for Higgs production in bottom fusion
}

\author{
Kemal J. Ozeren \\ Department of Physics and Astronomy, UCLA, \\ Los Angeles, CA 90095-1547, U.S.A. \\ Fachbereich C, Bergische Universität Wuppertal, \\ 42097 Wuppertal, Germany \\ E-mail: ozeren@physics.ucla.edu
}

ABSTRACT: We evaluate analytically the cross section for Higgs production plus one jet through bottom quark fusion. By considering the small $p_{T}$ limit we derive expressions for the resummation coefficients governing the structure of large logarithms, and compare these expressions with those available in the literature.

Keywords: NLO Computations, Higgs Physics

ARXIV EPRINT: 1010.2977 


\section{Contents}

1 Introduction 1

2 Notation 2

$\begin{array}{llr}3 & \text { NLO corrections } & 4\end{array}$

3.1 Real radiation 4

4 Results $\quad 6$

4.1 Checks 8

5 Small $p_{T}$ limit $\quad \mathbf{8}$

5.1 Fixed order expansion of the resummed formula 9

$\begin{array}{ll}5.2 \text { Extracting the resummation coefficients } & 10\end{array}$

6 Conclusion 12

\section{Introduction}

The Standard Model (SM) [1, 2] predicts the existence of a massive scalar particle known as the Higgs boson. Within this theory and its supersymmetric extensions [3], matter fields and gauge bosons acquire mass via the Higgs mechanism [4-6]. Although the Higgs remains undiscovered, experiments have placed restrictions on its mass [7, 8]. Supersymmetric theories require more than one Higgs boson. There are many more free parameters than in the SM, and the experimental constraints are correspondingly weaker $[9,10]$.

The Large Hadron Collider (LHC) is expected to find the Higgs boson if it exists. To achieve this, various production mechanisms must be considered. The relative utility of each depends strongly on the Higgs' mass and couplings. While in the SM gluon fusion is by far the largest contribution to the total cross section, in SUSY theories with large $\tan \beta$ bottom quark fusion can dominate, due to the enhanced $b \bar{b} H$ Yukawa coupling [11-13]. Reviews can be found in refs. $[14,15]$. If we assume that the proton is composed only of the four lightest quarks and the gluon, the so called four flavour scheme (4FS), then the dominant leading order diagram for this process is that shown in figure 1(a). Integration of the phase space leads to divergences arising from the kinematical region where one or both bottom quarks are collinear to the initial state partons. The bottom's mass $m_{b}$ regulates these divergences, but they still leave traces in the form of large logarithms $\ln \left(m_{b}^{2} / m_{\mathrm{H}}^{2}\right)$. Such logarithms jeopordise the convergence of the perturbative series, so ideally one would like to resum them. This can be achieved by introducing bottom quark PDFs. In this five flavour scheme $(5 \mathrm{FS})[16,17]$ the $b$ quark can appear in the initial state, and so the leading order process is changed to that appearing in figure 1(b). One sets the $b$ 


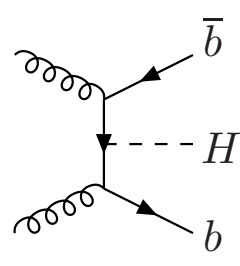

(a)

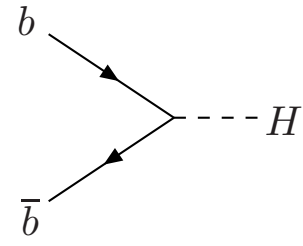

(b)

Figure 1. Diagrams for Higgs production through bottom fusion in the (a) four and (b) five flavour schemes.

quark mass to zero in this case. Results obtained in either scheme should be the same, although when truncating at a finite order there will be differences, formally of higher order in $\alpha_{s}$. Despite this, it was found that the inclusive cross sections in the 4FS and the 5FS differ by roughly a factor of five when evaluated at $\mu_{\mathrm{F}}=\mu_{\mathrm{R}}=m_{\mathrm{H}}$, where $\mu_{\mathrm{F}} / \mu_{\mathrm{R}}$ is the factorisation/renormalisation scale. This remains true also at NLO QCD which was calculated for the 5FS in refs. [18, 19], and for the 4FS in refs. [20, 21]. It was thus proposed in refs. $[19,22-24]$ that when using the five flavour scheme the appropriate central scale is $m_{H} / 4$. Indeed, the NNLO result [25] in the 5FS seems to confirm this choice.

Higgs production in association with one or more jets [26] has also received much attention. In the case of gluon fusion the leading order cross section is known, including the full top and bottom mass dependence, in both the SM [26-28] and MSSM [28]. The NLO corrections are known only in the heavy-top limit [29-31]. As far as the MSSM is concerned, one expects that to a very good approximation one can simply replace the effective $g g H$ coupling of the Standard Model with its MSSM value [32-34]. However, as we have stressed, for large $\tan \beta$ one must also include the bottom fusion contribution. That is the subject of this paper.

The bottom fusion contribution to $H+$ jet production has been considered for the case in which a final state $b$ quark is tagged [35]. This is a useful observable because one can measure the $b \bar{b} H$ Yukawa coupling directly. Without $b$ tagging, this process is a contribution to the total $H+$ jet cross section, and must be considered alongside gluon fusion. For this case various distributions at NLO have been presented [36], based on Catani-Seymour subtraction. In this paper we give the same cross section analytically. As well as providing a very strong check on the results of ref. [36], our results allow us to analytically take the $p_{T} \rightarrow 0$ limit, and thus derive expressions for the resummation coefficients which govern the structure of large logarithms. This will be described in section 5 .

\section{Notation}

We are interested in the transverse momentum distribution of Higgs bosons arising from the scattering of two hadrons $h_{1}$ and $h_{2}$ at centre of mass energy $\sqrt{S}$. In particular, we consider in this paper only that part of the cross section proportional to the $H b \bar{b}$ Yukawa coupling. The $b$-quark mass is set to zero everywhere except in this coupling. For a Higgs 
boson with tranverse momentum $p_{T}$ and rapidity $y$, the cross section is

$$
\frac{\mathrm{d} \sigma}{\mathrm{d} p_{T}^{2} \mathrm{~d} y}=\sum_{i j} \frac{1}{1+\delta_{i j}} \int \mathrm{d} x_{1} \int \mathrm{d} x_{2} f_{i / h_{1}}\left(x_{1}, \mu_{\mathrm{F}}\right) f_{j / h_{2}}\left(x_{2}, \mu_{\mathrm{F}}\right) \frac{\mathrm{d} \hat{\sigma}_{i j}}{\mathrm{~d} p_{T}^{2} \mathrm{~d} y},
$$

where $f_{i / h_{1}}\left(x_{1}, \mu_{\mathrm{F}}\right)$ is the parton density for finding a parton $i$ in hadron $h_{1}$. We expand the partonic cross section appearing on the right hand side in powers of the strong coupling constant $\alpha_{s}\left(\mu_{\mathrm{R}}\right)$,

$$
\frac{\mathrm{d} \hat{\sigma}_{i j}}{\mathrm{~d} p_{T}^{2} \mathrm{~d} y}=\frac{\pi}{8} \frac{m_{b}^{2}}{\mathcal{V}^{2}} \frac{1}{\hat{s}} \frac{1}{\mathcal{C}_{i j}}\left[\frac{\alpha_{s}\left(\mu_{\mathrm{R}}\right)}{2 \pi} G_{i j}^{(1)}\left(\mu_{R}\right)+\left(\frac{\alpha_{s}\left(\mu_{\mathrm{R}}\right)}{2 \pi}\right)^{2} G_{i j}^{(2)}\left(\mu_{R}\right)+\cdots\right],
$$

where $\mathcal{V}=246 \mathrm{GeV}$ is the vacuum expectation value of the Higgs field, $m_{b}$ is the bottom quark mass, $\mathcal{C}_{i j}$ is a colour averaging factor $\left(\mathcal{C}_{i j}=9\right.$ for quark-quark scattering, etc. $)$ and the dots stand for higher terms in the $\alpha_{s}$ expansion. Although the partonic cross section itself is not a function of the renormalisation scale $\mu_{\mathrm{R}}$, its expansion coefficients $G_{i j}^{(n)}$ are, so that truncating at any finite order of perturbation theory leads to an unphysical $\mu_{\mathrm{R}}$ dependence of the cross section. Reducing this unphysical scale dependence is one of the primary motivations for calculating higher order QCD corrections.

We denote the four momenta of the incoming hadrons as $P_{1}$ and $P_{2}$, while those of the colliding partons are $p_{1}=x_{1} P_{1}$ and $p_{2}=x_{2} P_{2}$. The mass, transverse momentum and rapidity of the Higgs boson are written $m_{\mathrm{H}}, p_{T}$ and $y$ respectively. Momentum conservation implies

$$
p_{1}+p_{2}=Q+p_{H},
$$

where $Q$ represents the total momentum of the final state QCD partons, of which there can be either one or two. These we will label $p_{3}$ and $p_{4}$. Our results for the coefficients $G_{i j}^{(n)}$ will be given in terms of the following partonic invariants

$$
\begin{aligned}
s & =\left(p_{1}+p_{2}\right)^{2}, \\
u & =\left(p_{1}-Q\right)^{2}, \\
t & =\left(p_{2}-Q\right)^{2},
\end{aligned}
$$

in terms of which momentum conservation imposes the constraint

$$
s+u+t=m_{\mathrm{H}}^{2}+Q^{2} .
$$

In terms of these variables the transverse momentum of the Higgs satisfies

$$
p_{T}^{2}=\frac{u t-m_{\mathrm{H}}^{2} Q^{2}}{s} .
$$

It is also useful to define

$$
\begin{aligned}
S_{u} & =u-Q^{2}, \\
S_{t} & =t-Q^{2}, \\
m_{T}^{2} & =m_{\mathrm{H}}^{2}+p_{T}^{2}, \\
v & =\frac{p_{T}^{2}}{Q^{2}+p_{T}^{2}} .
\end{aligned}
$$



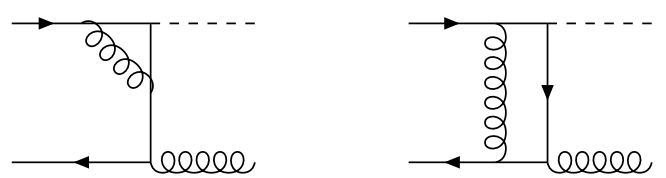

Figure 2. Examples of one loop diagrams at NLO.

At leading order $\left(\mathcal{O}\left(\alpha_{s}\right)\right)$ there are two contributing channels. We find for the corresponding coefficients $G_{i j}^{(1)}=g_{i j} \delta\left(Q^{2}\right)$ with,

$$
\begin{aligned}
& g_{b \bar{b}}=4 C_{F} C_{A} \frac{\left(s^{2}+m_{\mathrm{H}}^{4}\right)}{u t}, \\
& g_{b g}=4 C_{F} C_{A} \frac{\left(u^{2}+m_{\mathrm{H}}^{4}\right)}{-s t},
\end{aligned}
$$

where $C_{F}=\frac{4}{3}$ and $C_{A}=3$. We will discuss how to integrate these expressions over the momentum fractions $x_{1,2}$ in section 4 .

\section{$3 \quad$ NLO corrections}

The $\mathcal{O}\left(\alpha_{s}^{2}\right)$ corrections, which form the coefficient function $G_{i j}^{(2)}$, receive three different types of contribution: the one loop virtual corrections to the leading order processes, for which some sample diagrams are given in figure 2, the mass factorisation pieces, arising from the definition of the parton densities at NLO, and finally the real radiation contribution. Each of these pieces is divergent in four dimensions, so that in practice a regularisation procedure is required. We use conventional dimensional regularisation (CDR), working in $d=4-2 \epsilon$ dimensions, so that the divergences manifest themselves as poles in the parameter $\epsilon$. For infrared safe observables these poles cancel, and at the end of the calculation we may safely take the limit $d \rightarrow 4$.

The one loop amplitude for $b \bar{b} \rightarrow H g$ was given in ref. [35], and we have independently checked the result. We also require $b g \rightarrow H b$, which can be obtained by crossing. The virtual parts contain ultraviolet divergences, which we remove by renormalising $\alpha_{s}$ and $m_{b}$ in the $\overline{\mathrm{MS}}$ scheme.

At NLO there are also additional channels to consider, beyond those which contribute at LO. In our case these are $g g, b q, b b$ and $q \bar{q}$. Here $q$ represents one of the $u, d, s$ or $c$ quarks, and it is understood that the charge conjugated processes are also included. Because these channels do not contribute at leading order, none of them have a one loop correction. However, with the exception of $q \bar{q}$, they do require mass factorisation to remove collinear poles, and so must be regularised just as in the case of $b \bar{b}$ and $b g$.

\subsection{Real radiation}

We have evaluated the amplitudes for the Higgs plus two parton processes using FORM [37]. Sample diagrams are shown in figure 3. They are expressed in terms of the invariants $s_{i j}=\left(p_{i}+p_{j}\right)^{2}$ and $s_{i j k}=\left(p_{i}+p_{j}+p_{k}\right)^{2}$. Note that we must retain the $\mathcal{O}(\epsilon)$ pieces of the 

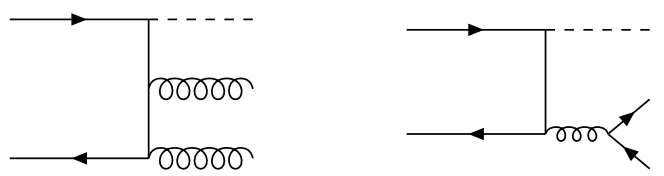

Figure 3. Examples of real emission diagrams at NLO. The initial state lines are bottom quarks, but the final state quarks can be of any flavour.

amplitudes, because integration over the three body phase space can generate poles in $\epsilon$. As a check one may verify that gauge invariance holds. We have also used MADGRAPH [38] to check the amplitudes in the $\epsilon \rightarrow 0$ limit.

We write the three body phase space factor as

$$
\mathrm{d} \Gamma_{3}=\left(\frac{4 \pi \mu^{2}}{Q^{2}}\right)^{\epsilon}\left(\frac{4 \pi \mu^{2}}{p_{T}^{2}}\right)^{\epsilon} \frac{1}{(4 \pi)^{2}} \frac{1}{\Gamma(1-2 \epsilon)} \frac{\mathrm{d} \Omega}{8 \pi} \mathrm{d} p_{T}^{2} \mathrm{~d} y,
$$

and integrate analytically over the angular factor $\mathrm{d} \Omega$, given by

$$
\int \mathrm{d} \Omega=\frac{1}{2 \pi} \int_{0}^{\pi} \sin ^{1-2 \epsilon} \theta \mathrm{d} \theta \int_{0}^{\pi} \sin ^{-2 \epsilon} \phi \mathrm{d} \phi .
$$

It is useful to work in the $Q$ rest frame. We can then obtain expressions [30] for the invariants in terms of angles and energies. To perform the integration we first make use of momentum conservation and numerous partial fraction identities to ensure that each term in the amplitude squared contains at most two invariants that depend on the angles $\theta$ and $\phi$. These invariants are $s_{13}, s_{14}, s_{23}, s_{24}, s_{123}$ and $s_{124}$. For example, the relation

$$
\frac{1}{s_{13} s_{14}}=\frac{1}{S_{u}}\left(\frac{1}{s_{13}}+\frac{1}{s_{14}}\right)
$$

reduces the number of angle dependent factors from two to one. Once this decomposition is achieved the integrals over $\mathrm{d} \Omega$ are of two types ( $m$ and $n$ are integers):

$$
\begin{aligned}
& \int d \Omega s_{23}^{-m} s_{13}^{-n}, \\
& \int d \Omega s_{123}^{-m} s_{13}^{-n} .
\end{aligned}
$$

A closed form result, valid for abritrary $\epsilon$, for integrals of the first type is given in ref. [39]. The integrals of the second type are given as expansions in $\epsilon$ in ref. [40]. We have used these results directly, supplementing them where necessary with extra $\mathcal{O}(\epsilon)$ terms.

As the integration over the momentum fractions $x_{1,2}$ is performed, divergences appear in the small $Q^{2}$ region due to terms with $1 / Q^{2}$ factors. These divergences are regulated ${ }^{1}$ by the $Q^{-2 \epsilon}$ factor appearing eq.(3.1). To expose the corresponding poles in $\epsilon$ we use the distribution relation

$$
\left(Q^{2}\right)^{-1-\epsilon} \rightarrow-\frac{1}{\epsilon} \delta\left(Q^{2}\right) A^{\epsilon}+\left(\frac{1}{Q^{2}}\right)_{+}-\epsilon\left(\frac{\ln Q^{2}}{Q^{2}}\right)_{+}+\mathcal{O}\left(\epsilon^{2}\right)
$$

\footnotetext{
${ }^{1}$ Strictly speaking, we mean 'made integrable'.
} 
where $A$ is the maximum value of $Q^{2}$. The plus distributions above will appear in our final results. They are defined by

$$
\int_{0}^{A} \mathrm{~d} Q^{2} f\left(Q^{2}\right)\left[g\left(Q^{2}\right)\right]_{+}=\int_{0}^{A} \mathrm{~d} Q^{2}\left[f\left(Q^{2}\right)-f(0)\right] g\left(Q^{2}\right)
$$

There are still divergences in the small $p_{T}$ region. These are regulated by the $p_{T}^{-2 \epsilon}$ factor appearing in eq.(3.1). In principle we could make use of a distribution relation similar to that above to expose the divergences as poles in $\epsilon$. We would then need to add the two loop corrections to the process $b \bar{b} \rightarrow H$, as well as some extra terms arising from mass factorisation. The result would be a NNLO result for the differential cross section of the process $b \bar{b} \rightarrow H$. We do not take this extra step, which is difficult to achieve in practice, so that in our numerical results we must avoid the small $p_{T}$ region. Our results therefore constitute a NLO result for the process $b \bar{b} \rightarrow H+$ jet. We will discuss the behaviour of the cross section in the $p_{T} \rightarrow 0$ limit in more detail in section 5 .

\section{Results}

Our result for the NLO $b \bar{b}$ coefficient function takes the form

$$
G_{b \bar{b}}^{(2)}=D_{b \bar{b}} \delta\left(Q^{2}\right)+E_{b \bar{b}}\left(\frac{1}{Q^{2}}\right)_{+}+F_{b \bar{b}}\left(\frac{1}{Q^{2}} \ln \frac{Q^{2}}{m_{\mathrm{H}}^{2}}\right)_{+}+H_{b \bar{b}}
$$

We define

$$
\begin{aligned}
\kappa & =\frac{1}{2}\left(m_{\mathrm{H}}^{2}+s-Q^{2}\right), \\
\lambda^{2} & =\kappa^{2}-m_{\mathrm{H}}^{2} s, \\
x & =\frac{\kappa+\lambda}{\kappa-\lambda},
\end{aligned}
$$

and introduce the following convenient logarithm abbreviations.

$$
\begin{aligned}
& L_{s}=\ln \frac{s}{m_{\mathrm{H}}^{2}}, \quad L_{u}=\ln \frac{-u}{m_{\mathrm{H}}^{2}}, \quad L_{t}=\ln \frac{-t}{m_{\mathrm{H}}^{2}}, \\
& l_{F}=\ln \frac{\mu_{\mathrm{F}}^{2}}{m_{\mathrm{H}}^{2}}, \quad l_{R}=\ln \frac{\mu_{\mathrm{R}}^{2}}{m_{\mathrm{H}}^{2}}, \quad L_{A}=\ln \frac{A}{m_{\mathrm{H}}^{2}} \text {. }
\end{aligned}
$$

We give here the $D_{b \bar{b}}, E_{b \bar{b}}$ and $F_{b \bar{b}}$ coefficients. The expressions for $H_{b \bar{b}}$ are very large, so we do not reproduce them here. Results for all the above coefficients, for all channels, are 
available from the author on request.

$$
\begin{aligned}
D_{b \bar{b}}= & g_{b \bar{b}} C_{A}\left[-L_{A}^{2}-2 L_{A} L_{s}+2 L_{A} L_{t}+2 L_{A} L_{u}-\frac{11 L_{A}}{6}+\frac{11 l_{R}}{6}-L_{s}^{2}+2 L_{s} L_{t}\right. \\
& \left.+2 L_{s} L_{u}-L_{t}^{2}-2 L_{t} L_{u}-L_{u}^{2}-2 \mathrm{Li}_{2}\left(1-\frac{m_{\mathrm{H}}^{2}}{s}\right)+\frac{67}{18}\right] \\
& +g_{b \bar{b}} C_{F}\left[4 L_{A}^{2}+4 L_{A} L_{s}-4 L_{A} L_{t}-4 L_{A} L_{u}-4 L_{A} l_{F}+3 l_{R}+2 L_{s}^{2}\right. \\
& -4 L_{s} L_{t}-4 L_{s} L_{u}+L_{t}^{2}+2 L_{t} L_{u}+2 L_{t} l_{F}+L_{u}^{2}+2 L_{u} l_{F}-3 l_{F} \\
& +4 \operatorname{Li}_{2}\left(1-\frac{m_{\mathrm{H}}^{2}}{s}\right)+2 \operatorname{Li}_{2}\left(\frac{m_{\mathrm{H}}^{2}}{m_{\mathrm{H}}^{2}-t}\right)+2 \mathrm{Li}_{2}\left(\frac{m_{\mathrm{H}}^{2}}{m_{\mathrm{H}}^{2}-u}\right) \\
& \left.+\ln ^{2}\left(1-\frac{t}{m_{\mathrm{H}}^{2}}\right)+\ln { }^{2}\left(1-\frac{u}{m_{\mathrm{H}}^{2}}\right)+\frac{\pi^{2}}{3}-2\right] \\
& +\frac{2}{3} n_{f} T_{R} g_{b \bar{b}}\left[L_{A}-l_{R}-\frac{5}{3}\right]+4 m_{\mathrm{H}}^{2}\left(\frac{1}{u}+\frac{1}{t}\right) C_{F} C_{A}\left(C_{A}-C_{F}\right) \\
E_{b \bar{b}}= & \left.C_{A} g_{b \bar{b}}\left[\ln (1-v)+\ln \frac{S_{u}}{u}+\ln \frac{S_{t}}{t}+2 \ln \frac{Q^{2}+p_{T}^{2}}{m_{\mathrm{H}}^{2}}-L_{s}-\frac{11}{6}\right)\right] \\
& +C_{F} g_{b \bar{b}}\left[-2 \ln \frac{S_{u}}{u}-2 \ln \frac{S_{t}}{t}-4 l_{F}-4 \ln \frac{Q^{2}+p_{T}^{2}}{m_{\mathrm{H}}^{2}}+2 L_{s}\right]+\frac{2}{3} T_{R} n_{F} g_{b \bar{b}} \\
& +4 C_{F} C_{A}\left(C_{F}-\frac{C_{A}}{2}\right) \frac{\ln x}{\lambda}\left[2 \frac{m_{\mathrm{H}}^{4}}{t}+\frac{m_{\mathrm{H}}^{4}}{u}-2 \frac{m_{\mathrm{H}}^{2} u}{t}-m_{\mathrm{H}}^{2}+\frac{s^{2}}{u}-s+\frac{u^{2}}{t}+u\right] \\
F_{b \bar{b}}= & 8\left(C_{F}-\frac{C_{A}}{4}\right) g_{b \bar{b}} .
\end{aligned}
$$

Recall that $S_{u}, S_{t}$ and $v$ were defined in eq. (2.7), while $g_{b \bar{b}}$ was given in eq. (2.8). $\mathrm{Li}_{2}$ denotes the dilogarithm function. For QCD the colour factors take the values $C_{F}=\frac{4}{3}$, $C_{A}=3$ and $T_{R}=\frac{1}{2}$.

To integrate the distributions above over the PDFs, it is useful to arrange for $Q^{2}$ to be one of the integration variables. This is easily achieved [41], with the result that we can replace

$$
\int_{0}^{1} \mathrm{~d} x_{1} \int_{0}^{1} \mathrm{~d} x_{2} \theta\left(Q^{2}\right) \rightarrow \frac{1}{S} \int_{x_{+}}^{1} \frac{\mathrm{d} x_{1}}{x_{1}-x_{U}} \int_{0}^{A_{1}} \mathrm{~d} Q^{2}+\frac{1}{S} \int_{x_{-}}^{1} \frac{\mathrm{d} x_{2}}{x_{2}-x_{T}} \int_{0}^{A_{2}} \mathrm{~d} Q^{2},
$$

where

$$
\begin{aligned}
& x_{U}=\frac{m_{T}^{2}}{S} e^{y}, \\
& x_{T}=\frac{m_{T}^{2}}{S} e^{-y}, \\
& x_{ \pm}=\frac{m_{T}+p_{T}}{\sqrt{S}} e^{ \pm y}, \\
& A_{1}=x_{1}\left(1-x_{T}\right)-x_{U}+\frac{m_{\mathrm{H}}^{2}}{S}, \\
& A_{2}=x_{+}\left(x_{2}-x_{T}\right)-x_{U} x_{2}+\frac{m_{\mathrm{H}}^{2}}{S} .
\end{aligned}
$$


The expression for $A_{1}$ or $A_{2}$, as appropriate, should be substituted in eq. (4.6) in place of $A$. In this way one can obtain numerical results for the hadronic cross section. Phenomenological analyses have already been presented in ref. [36], so we do not repeat it here.

\subsection{Checks}

We have performed a number of checks on our results. Firstly, the dependence of the cross section on $\mu_{\mathrm{F}}$ and $\mu_{\mathrm{R}}$ can be predicted due to the requirement that physical observables must be independent of these scales. Our expressions for the perturbative coefficients $G_{i j}^{(n)}$ satisfy these constraints. Secondly, as we will discuss in the next section, the small $p_{T}$ behaviour can be compared to known resummed formulae.

The strongest check is a comparison with a Monte Carlo numerical code [36] based on the Catani Seymour [42] subtraction formalism. We find excellent agreement for all channels. In the case of the $q \bar{q}$ channel, one can also compare to the known total cross section [25] by numerically integrating eq.(2.1) over $p_{T}$ and $y$.

\section{$5 \quad$ Small $p_{T}$ limit}

For small $p_{T}$ the convergence of fixed order perturbation theory is spoiled at any finite order by terms of the form

$$
\frac{\ln ^{n} p_{T}^{2}}{p_{T}^{2}}, \quad n=0,1,2,3 .
$$

To obtain reliable physical predictions for observables in this region one must resum these enhanced terms. The method to perform this resummation is known. The result [43] is expressed as an integral over the impact parameter $b$,

$$
\frac{\mathrm{d} \sigma}{\mathrm{d} p_{T}^{2} \mathrm{~d} y}=\frac{m_{\mathrm{H}}^{2} \sigma_{0}\left(m_{\mathrm{H}}\right)}{2 S} \int_{0}^{\infty} b \mathrm{~d} b J_{0}\left(b p_{T}\right) W(b)
$$

where the bottom quark mass $m_{b}$, implicit in the prefactor $\sigma_{0}$, is evaluated at the scale $\mu_{R}=m_{\mathrm{H}}$. The Sudakov form factor $W(b)$ contains the large logarithms, and is defined as

$$
\begin{aligned}
W(b)= & \left(C_{b i}\left(\alpha_{s}\left(b_{0} / b\right)\right) \otimes f_{i}\right)\left(\bar{x}_{1}^{0} ; b / b_{0}\right)\left(C_{\bar{b} j}\left(\alpha_{s}\left(b_{0} / b\right)\right) \otimes f_{j}\right)\left(\bar{x}_{2}^{0} ; b / b_{0}\right) \\
& \times \exp \left\{-\int_{b_{0}^{2} / b^{2}}^{m_{\mathrm{H}}^{2}} \frac{\mathrm{d} q^{2}}{q^{2}}\left[A\left(\alpha_{s}(q)\right) \ln \frac{m_{\mathrm{H}}^{2}}{q^{2}}+B\left(\alpha_{s}(q)\right)\right]\right\}
\end{aligned}
$$

where $\otimes$ indicates convolution, partons $i, j$ are implicitly summed over and $b_{0}=2 e^{-\gamma_{E}}$, with $\gamma_{E}$ Euler's constant. The PDFs $f_{i}$ and $f_{j}$ are evaluated at the scale $b / b_{0}$, and

$$
\bar{x}_{1,2}^{0}=\frac{m_{\mathrm{H}}}{\sqrt{S}} e^{ \pm y} .
$$


The resummation coefficients $A, B$ and $C_{i j}$ can be expanded perturbatively,

$$
\begin{aligned}
A\left(\alpha_{s}\right) & =\sum_{n=1}^{\infty}\left(\frac{\alpha_{s}}{2 \pi}\right)^{n} A^{(n)}, \\
B\left(\alpha_{s}\right) & =\sum_{n=1}^{\infty}\left(\frac{\alpha_{s}}{2 \pi}\right)^{n} B^{(n)}, \\
C_{i j}\left(\alpha_{s}\right) & =\delta_{i j} \delta(1-z)+\sum_{n=1}^{\infty}\left(\frac{\alpha_{s}}{2 \pi}\right)^{n} C_{i j}^{(n)} .
\end{aligned}
$$

The coefficient $A^{(1)}$ controls the leading logarithmic (LL) terms, while $A^{(2)}, B^{(1)}$ and $C_{i j}^{(1)}$ give the next to leading logarithmic (NLL) terms, etc. They can be evaluated by performing a fixed order calculation and comparing to the resummed expression.

\subsection{Fixed order expansion of the resummed formula}

One cannot naively expand eq. (5.2) in powers of $\alpha_{s}$. Instead, we first integrate by parts (we can ignore the surface term) to obtain

$$
\frac{\mathrm{d} \sigma}{\mathrm{d} p_{T}^{2} \mathrm{~d} y}=-\frac{m_{\mathrm{H}}^{2} \sigma_{0}\left(m_{\mathrm{H}}\right)}{2 S} \frac{1}{p_{T}^{2}} \int_{0}^{\infty} b \mathrm{~d} b J_{1}(b) \frac{\mathrm{d} W(b)}{\mathrm{d} b} .
$$

With the $p_{T}^{2}$ pole now manifest, we can expand. We use the DGLAP equation to evolve the PDFs to an arbitrary scale $\mu_{\mathrm{F}}$. We also evolve the QCD coupling $\alpha_{s}$ and the bottom quark mass $m_{b}$ from their values at the scale $m_{\mathrm{H}}$ or $q$ to an arbitrary scale $\mu_{\mathrm{R}}$. The expanded resummed cross section is, using the notation of ref. [31],

$$
\left.\frac{\mathrm{d} \sigma}{\mathrm{d} p_{T}^{2} \mathrm{~d} y}\right|_{p_{T} \ll m}=\frac{\sigma_{0}}{S} \frac{m_{\mathrm{H}}^{2}}{p_{T}^{2}}\left[\sum_{m=1}^{2} \sum_{n=0}^{2 m-1}\left(\frac{\alpha_{s}}{2 \pi}\right)^{m}{ }_{m} C_{n}\left(\ln \frac{m_{\mathrm{H}}^{2}}{p_{T}^{2}}\right)^{n}+\mathcal{O}\left(\alpha_{s}^{3}\right)\right] .
$$


The coefficients ${ }_{m} C_{n}$ are related to the resummation coefficients as follows,

$$
\begin{aligned}
{ }_{1} C_{1}= & A^{(1)} f_{b}\left(\bar{x}_{1}^{0}\right) f_{\bar{b}}\left(\bar{x}_{2}^{0}\right), \\
{ }_{1} C_{0}= & B^{(1)} f_{b}\left(\bar{x}_{1}^{0}\right) f_{\bar{b}}\left(\bar{x}_{2}^{0}\right)+\left(P_{b i} \otimes f_{i}\right)\left(\bar{x}_{1}^{0}\right) f_{\bar{b}}\left(\bar{x}_{2}^{0}\right)+f_{b}\left(\bar{x}_{1}^{0}\right)\left(P_{\bar{b} i} \otimes f_{i}\right)\left(\bar{x}_{2}^{0}\right), \\
{ }_{2} C_{3}= & -\frac{1}{2}\left[A^{(1)}\right]^{2} f_{b}\left(\bar{x}_{1}^{0}\right) f_{\bar{b}}\left(\bar{x}_{2}^{0}\right), \\
{ }_{2} C_{2}= & -\frac{3}{2} A^{(1)}\left[\left(P_{b i} \otimes f_{i}\right)\left(\bar{x}_{1}^{0}\right) f_{\bar{b}}\left(\bar{x}_{2}^{0}\right)+f_{b}\left(\bar{x}_{1}^{0}\right)\left(P_{\bar{b} i} \otimes f_{i}\right)\left(\bar{x}_{2}^{0}\right)\right] \\
& +A^{(1)}\left[\beta_{0}-\frac{3}{2} B^{(1)}\right] f_{b}\left(\bar{x}_{1}^{0}\right) f_{\bar{b}}\left(\bar{x}_{2}^{0}\right) \\
{ }_{2} C_{1}= & {\left[\beta_{0}-2 B^{(1)}-A^{(1)} \ln \frac{\mu_{\mathrm{F}}^{2}}{m_{\mathrm{H}}^{2}}\right]\left(P_{b i} \otimes f_{i}\right)\left(\bar{x}_{1}^{0}\right) f_{\bar{b}}\left(\bar{x}_{2}^{0}\right)+A^{(1)}\left(C_{b i}^{(1)} \otimes f_{i}\right)\left(\bar{x}_{1}^{0}\right) f_{\bar{b}}\left(\bar{x}_{2}^{0}\right) } \\
& -\left(P_{b i} \otimes f_{i}\right)\left(\bar{x}_{1}^{0}\right)\left(P_{\bar{b} j} \otimes f_{j}\right)\left(\bar{x}_{2}^{0}\right)-\left(P_{b i} \otimes P_{i j} \otimes f_{j}\right)\left(\bar{x}_{1}^{0}\right) f_{\bar{b}}\left(\bar{x}_{2}^{0}\right) \\
& -\frac{1}{2}\left[\left[B^{(1)}\right]^{2}-A^{(2)}-\beta_{0} B^{(1)}-\beta_{0}^{\prime} A^{(1)} \ln \frac{\mu_{\mathrm{R}}^{2}}{m_{\mathrm{H}}^{2}}\right] f_{b}\left(\bar{x}_{1}^{0}\right) f_{\bar{b}}\left(\bar{x}_{2}^{0}\right) \\
& +\left\{b, \bar{x}_{1}^{0} \leftrightarrow \bar{b}, \bar{x}_{2}^{0}\right\} \\
{ }_{2} C_{0}= & -\left[\left(P_{b i} \otimes f_{i}\right)\left(\bar{x}_{1}^{0}\right)\left(P_{\bar{b} j} \otimes f_{j}\right)\left(\bar{x}_{2}^{0}\right)+\left(P_{b i} \otimes P_{i j} \otimes f_{j}\right)\left(\bar{x}_{1}^{0}\right) f_{\bar{b}}\left(\bar{x}_{2}^{0}\right)\right] \ln \frac{\mu_{\mathrm{F}}^{2}}{m_{\mathrm{H}}^{2}} \\
& +\left[\beta_{0}^{\prime} \ln \frac{\mu_{\mathrm{R}}^{2}}{m_{\mathrm{H}}^{2}}-B^{(1)} \ln \frac{\mu_{\mathrm{F}}^{2}}{m_{\mathrm{H}}^{2}}\right]\left(P_{b i} \otimes f_{i}\right)\left(\bar{x}_{1}^{0}\right) f_{\bar{b}}\left(\bar{x}_{2}^{0}\right) \\
& +\left(C_{b i}^{(1)} \otimes f_{i}\right)\left(\bar{x}_{1}^{0}\right)\left(P_{\bar{b} j} \otimes f_{j}\right)\left(\bar{x}_{2}^{0}\right)+\left(C_{b i}^{(1)} \otimes P_{i j} \otimes f_{j}\right)\left(\bar{x}_{1}^{0}\right) f_{\bar{b}}\left(\bar{x}_{2}^{0}\right) \\
& +\left[\zeta_{3}\left[A^{(1)}\right]^{2}+\frac{1}{2} B^{(2)}+\frac{1}{2} \beta_{0}^{\prime} B^{(1)} \ln \frac{\mu_{\mathrm{R}}^{2}}{m_{\mathrm{H}}^{2}}\right] f_{b}\left(\bar{x}_{1}^{0}\right) f_{\bar{b}}\left(\bar{x}_{2}^{0}\right) \\
& +\left(B^{(1)}-\beta_{0}\right)\left(C_{b i}^{(1)} \otimes f_{i}\right)\left(\bar{x}_{1}^{0}\right) f_{\bar{b}}\left(\bar{x}_{2}^{0}\right)+\left(P_{b i}^{(2)} \otimes f_{i}\right)\left(\bar{x}_{1}^{0}\right) f_{\bar{b}}\left(\bar{x}_{2}^{0}\right) \\
& +\left\{b, \bar{x}_{1}^{0} \leftrightarrow \bar{b}, \bar{x}_{2}^{0}\right\} . \\
&
\end{aligned}
$$

where $\zeta_{n}$ is the Riemann $\zeta$-function $\left(\zeta_{3}=1.202 \ldots\right)$ and $\beta_{0}=\left(11 C_{A}-4 T_{R} n_{f}\right) / 6$. The corresponding expansions for Drell Yan production [44] and Higgs production through gluon fusion [31] have been presented before. Our case is slightly different to each of these due to the $\mu_{\mathrm{R}}$ dependence of the $H b \bar{b}$ Yukawa coupling. This is reflected in the modified beta coefficient $\beta_{0}^{\prime}=\beta_{0}+3 C_{F}$. The two loop splitting function $P_{b \bar{b}}^{(2)}$ can be extracted from the results of $[45,46]$.

\subsection{Extracting the resummation coefficients}

We have checked analytically that in the limit of small Higgs transverse momentum our results reproduce the resummed result, when the latter is expanded to the appropriate order in $\alpha_{s}$. Taking the limit analytically requires great care, because as well as explicitly singular terms appearing in our results, some logarithms of $p_{T}$ appear only upon integration over the momentum fractions $x_{1,2}$.

By comparing with eq. (5.9) we can derive the values of the resummation coefficients 


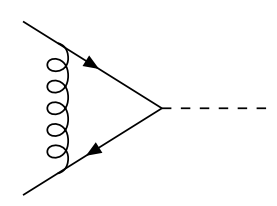

Figure 4. Diagram controlling the process-dependent part of the NNLL resummation coefficient $B^{(2)}$, as described in ref. [48].

$A, B$ and $C_{i j}$. For the universal coefficients we find the expected values,

$$
\begin{aligned}
& A^{(1)}=2 C_{F}, \\
& A^{(2)}=2 C_{F}\left(\frac{67}{18} C_{A}-\frac{10}{9} n_{f} T_{R}-\frac{\pi^{2}}{6} C_{A}\right), \\
& B^{(1)}=-3 C_{F} .
\end{aligned}
$$

For the process-specific coefficients we have

$$
\begin{aligned}
B^{(2)} & =C_{F}^{2}\left(-\frac{3}{4}+\pi^{2}-12 \zeta_{3}\right)+C_{F} C_{A}\left(-\frac{61}{12}+\frac{11}{9} \pi^{2}+6 \zeta_{3}\right)+C_{F} T_{R} n_{f}\left(\frac{5}{3}-\frac{4}{9} \pi^{2}\right), \\
C_{b \bar{b}}^{(1)} & =C_{F}\left[1-x+\left(\frac{\pi^{2}}{2}-1\right) \delta(1-x)\right], \\
C_{b g}^{(1)} & =2 T_{R} x(1-x) .
\end{aligned}
$$

The expressions for $C_{i j}^{(1)}$ match those given in ref. [47]. Our result for $B^{(2)}$ is the first direct calculation of this quantity. It has been shown [48] that $B^{(2)}$ can be split into universal and process dependent parts, and that furthermore, the process dependent part is directly related to the finite part $\mathcal{A}$ of the one loop correction to the leading order process, which in our case is $b \bar{b} \rightarrow H$. For quark initiated processes, the relationship is expressed as

$$
B^{(2)}=-2 \gamma^{(2)}+\beta_{0}\left(\frac{2}{3} C_{F} \pi^{2}+\mathcal{A}\right)
$$

where $\gamma^{(2)}$ is the coefficient of $\delta(1-z)$ in the two loop splitting function $P_{q \bar{q}}^{(2)}(z)$, given by

$$
\gamma^{(2)}=C_{F}^{2}\left(\frac{3}{8}-\frac{\pi^{2}}{2}+6 \zeta_{3}\right)+C_{F} C_{A}\left(\frac{17}{24}+\frac{11}{18} \pi^{2}-3 \zeta_{3}\right)-C_{F} n_{F} T_{R}\left(\frac{1}{6}+\frac{2}{9} \pi^{2}\right) .
$$

It is straightforward to evaluate the one loop correction to $b \bar{b} \rightarrow H$ (the single contributing diagram is shown in figure 4), from which we find

$$
\mathcal{A}=C_{F}\left(-2+\frac{2}{3} \pi^{2}\right)
$$

Substituting this into eq. (5.14) yields the same expression for $B^{(2)}$ as we have derived from our analytic form of the cross section. 


\section{Conclusion}

We have described the analytic calculation of the cross section $b \bar{b} \rightarrow H+$ jet. The partonic cross section is a distribution in $Q^{2}$, the invariant mass of the final state QCD partons. The results agree numerically with an implementation based on Catani-Seymour subtraction [36].

By taking the limit of small Higgs transverse momentum, we have evaluated the resummation coefficients that govern the structure of large logarithms, including the NNLL coefficient $B^{(2)}$. This is the first direct calculation of this quantity for this process. It agrees with the general expression [48] relating $B^{(2)}$ to a one loop amplitude.

As well as being of phenomenological interest in their own right (numerical analyses have already been presented [36]), our results can form part of a differential NNLO calculation, perhaps along the lines of ref. [49].

\section{Acknowledgments}

I am grateful to Robert Harlander for many productive discussions throughout the project, and also to Massimiliano Grazzini for useful comments on the manuscript. This research was supported by the US Department of Energy under contract DE-FG03-91ER40662.

Open Access. This article is distributed under the terms of the Creative Commons Attribution Noncommercial License which permits any noncommercial use, distribution, and reproduction in any medium, provided the original author(s) and source are credited.

\section{References}

[1] S.L. Glashow, Partial Symmetries of Weak Interactions, Nucl. Phys. 22 (1961) 579 [SPIRES].

[2] S. Weinberg, A Model of Leptons, Phys. Rev. Lett. 19 (1967) 1264 [SPIRES].

[3] H.P. Nilles, Supersymmetry, Supergravity and Particle Physics, Phys. Rept. 110 (1984) 1 [SPIRES].

[4] G.S. Guralnik, C.R. Hagen and T.W.B. Kibble, Global Conservation Laws and Massless Particles, Phys. Rev. Lett. 13 (1964) 585 [SPIRES].

[5] F. Englert and R. Brout, Broken Symmetry and the Mass of Gauge Vector Mesons, Phys. Rev. Lett. 13 (1964) 321 [SPIRES].

[6] P.W. Higgs, Broken Symmetries and the Masses of Gauge Bosons, Phys. Rev. Lett. 13 (1964) 508 [SPIRES].

[7] LEP Working Group For Higgs boson searches collaboration, R. Barate et al., Search for the standard model Higgs boson at LEP, Phys. Lett. B 565 (2003) 61 [hep-ex/0306033] [SPIRES].

[8] CDF AND D0 collaboration, T. Aaltonen et al., Combination of Tevatron searches for the standard model Higgs boson in the W+W- decay mode, Phys. Rev. Lett. 104 (2010) 061802 [arXiv: 1001.4162] [SPIRES]. 
[9] ALEPH collaboration, S. Schael et al., Search for neutral MSSM Higgs bosons at LEP, Eur. Phys. J. C 47 (2006) 547 [hep-ex/0602042] [SPIRES].

[10] Tevatron New Phenomena \& Higgs Working Group collaboration, D. Benjamin et al., Combined CDF and DO upper limits on MSSM Higgs boson production in tau-tau final states with up to 2.2 fb-1, arXiv: 1003.3363 [SPIRES].

[11] A. Belyaev, A. Blum, R.S. Chivukula and E.H. Simmons, The meaning of Higgs: tau+ tauand gamma gamma at the Tevatron and the LHC, Phys. Rev. D 72 (2005) 055022 [hep-ph/0506086] [SPIRES].

[12] A. Belyaev, J. Pumplin, W.-K. Tung and C.P. Yuan, Uncertainties of the inclusive Higgs production cross section at the Tevatron and the LHC, JHEP 01 (2006) 069 [hep-ph/0508222] [SPIRES].

[13] O. Brein, Electroweak and Bottom Quark Contributions to Higgs Boson plus Jet Production, Phys. Rev. D 81 (2010) 093006 [arXiv: 1003.4438] [SPIRES].

[14] A. Djouadi, The Anatomy of electro-weak symmetry breaking. I: The Higgs boson in the standard model, Phys. Rept. 457 (2008) 1 [hep-ph/0503172] [SPIRES].

[15] A. Djouadi, The Anatomy of electro-weak symmetry breaking. II. The Higgs bosons in the minimal supersymmetric model, Phys. Rept. 459 (2008) 1 [hep-ph/0503173] [SPIRES].

[16] R.M. Barnett, H.E. Haber and D.E. Soper, Ultraheavy Particle Production from Heavy Partons at Hadron Colliders, Nucl. Phys. B 306 (1988) 697 [SPIRES].

[17] D.A. Dicus and S. Willenbrock, Higgs Boson Production from Heavy Quark Fusion, Phys. Rev. D 39 (1989) 751 [SPIRES].

[18] D. Dicus, T. Stelzer, Z. Sullivan and S. Willenbrock, Higgs boson production in association with bottom quarks at next-to-leading order, Phys. Rev. D 59 (1999) 094016 [hep-ph/9811492] [SPIRES].

[19] F. Maltoni, Z. Sullivan and S. Willenbrock, Higgs-boson production via bottom-quark fusion, Phys. Rev. D 67 (2003) 093005 [hep-ph/0301033] [SPIRES].

[20] S. Dittmaier, M. Krämer and M. Spira, Higgs radiation off bottom quarks at the Tevatron and the LHC, Phys. Rev. D 70 (2004) 074010 [hep-ph/0309204] [SPIRES].

[21] S. Dawson, C.B. Jackson, L. Reina and D. Wackeroth, Exclusive Higgs boson production with bottom quarks at hadron colliders, Phys. Rev. D 69 (2004) 074027 [hep-ph/0311067] [SPIRES].

[22] D.L. Rainwater, M. Spira and D. Zeppenfeld, Higgs boson production at hadron colliders: Signal and background processes, hep-ph/0203187 [SPIRES].

[23] T. Plehn, Charged Higgs boson production in bottom gluon fusion, Phys. Rev. D 67 (2003) 014018 [hep-ph/0206121] [SPIRES].

[24] E. Boos and T. Plehn, Higgs-boson production induced by bottom quarks, Phys. Rev. D 69 (2004) 094005 [hep-ph/0304034] [SPIRES].

[25] R.V. Harlander and W.B. Kilgore, Higgs boson production in bottom quark fusion at next-tonext-to-leading order, Phys. Rev. D 68 (2003) 013001 [hep-ph/0304035] [SPIRES].

[26] R.K. Ellis, I. Hinchliffe, M. Soldate and J.J. van der Bij, Higgs Decay to tau+ tau-: A Possible Signature of Intermediate Mass Higgs Bosons at the SSC, Nucl. Phys. B 297 (1988) 221 [SPIRES]. 
[27] B. Field, S. Dawson and J. Smith, Scalar and pseudoscalar Higgs boson plus one jet production at the LHC and Tevatron, Phys. Rev. D 69 (2004) 074013 [hep-ph/0311199] [SPIRES].

[28] O. Brein and W. Hollik, MSSM Higgs bosons associated with high-p $p_{T}$ jets at hadron colliders, Phys. Rev. D 68 (2003) 095006 [hep-ph/0305321] [SPIRES].

[29] D. de Florian, M. Grazzini and Z. Kunszt, Higgs production with large transverse momentum in hadronic collisions at next-to-leading order, Phys. Rev. Lett. 82 (1999) 5209 [hep-ph/9902483] [SPIRES].

[30] V. Ravindran, J. Smith and W.L. Van Neerven, Next-to-leading order QCD corrections to differential distributions of Higgs boson production in hadron hadron collisions, Nucl. Phys. B 634 (2002) 247 [hep-ph/0201114] [SPIRES].

[31] C.J. Glosser and C.R. Schmidt, Next-to-leading corrections to the Higgs boson transverse momentum spectrum in gluon fusion, JHEP 12 (2002) 016 [hep-ph/0209248] [SPIRES].

[32] R.V. Harlander and M. Steinhauser, Hadronic Higgs Production and Decay in Supersymmetry at Next-to-Leading Order, Phys. Lett. B 574 (2003) 258 [hep-ph/0307346] [SPIRES].

[33] R.V. Harlander and M. Steinhauser, Supersymmetric Higgs production in gluon fusion at next- to-leading order, JHEP 09 (2004) 066 [hep-ph/0409010] [SPIRES].

[34] G. Degrassi and P. Slavich, On the NLO QCD corrections to Higgs production and decay in the MSSM, Nucl. Phys. B 805 (2008) 267 [arXiv:0806.1495] [SPIRES].

[35] J.M. Campbell, R.K. Ellis, F. Maltoni and S. Willenbrock, Higgs boson production in association with a single bottom quark, Phys. Rev. D 67 (2003) 095002 [hep-ph/0204093] [SPIRES].

[36] R.V. Harlander, K.J. Ozeren and M. Wiesemann, Higgs plus jet production in bottom quark annihilation at next-to-leading order, Phys. Lett. B 693 (2010) 269 [arXiv:1007.5411] [SPIRES].

[37] J.A.M. Vermaseren, New features of FORM, math-ph/0010025 [SPIRES].

[38] T. Stelzer and W.F. Long, Automatic generation of tree level helicity amplitudes, Comput. Phys. Commun. 81 (1994) 357 [hep-ph/9401258] [SPIRES].

[39] W.L. van Neerven, Dimensional Regularization Of Mass And Infrared Singularities In Two Loop On-Shell Vertex Functions, Nucl. Phys. B 268 (1986) 453 [SPIRES].

[40] W. Beenakker, H. Kuijf, W.L. van Neerven and J. Smith, QCD Corrections to Heavy Quark Production in p $\bar{p}$ Collisions, Phys. Rev. D 40 (1989) 54 [SPIRES].

[41] C.J. Glosser, Higgs boson production in hadron hadron colliders, hep-ph/0201054 [SPIRES].

[42] S. Catani and M.H. Seymour, A general algorithm for calculating jet cross sections in NLO QCD, Nucl. Phys. B 485 (1997) 291 [hep-ph/9605323] [SPIRES].

[43] J.C. Collins, D.E. Soper and G.F. Sterman, Transverse Momentum Distribution in Drell-Yan Pair and $W$ and $Z$ Boson Production, Nucl. Phys. B 250 (1985) 199 [SPIRES].

[44] P.B. Arnold and R.P. Kauffman, $W$ and $Z$ production at next-to-leading order: From large q(t) to small, Nucl. Phys. B 349 (1991) 381 [SPIRES].

[45] G. Curci, W. Furmanski and R. Petronzio, Evolution of Parton Densities Beyond Leading Order: The Nonsinglet Case, Nucl. Phys. B 175 (1980) 27 [SPIRES]. 
[46] W. Furmanski and R. Petronzio, Singlet Parton Densities Beyond Leading Order, Phys. Lett. B 97 (1980) 437 [SPIRES].

[47] A. Belyaev, P.M. Nadolsky and C.P. Yuan, Transverse momentum resummation for Higgs boson produced via bb-bar fusion at hadron colliders, JHEP 04 (2006) 004 [hep-ph/0509100] [SPIRES].

[48] D. de Florian and M. Grazzini, The structure of large logarithmic corrections at small transverse momentum in hadronic collisions, Nucl. Phys. B 616 (2001) 247 [hep-ph/0108273] [SPIRES].

[49] S. Catani, L. Cieri, G. Ferrera, D. de Florian and M. Grazzini, Vector boson production at hadron colliders: a fully exclusive $Q C D$ calculation at $N N L O$,

Phys. Rev. Lett. 103 (2009) 082001 [arXiv:0903.2120] [SPIRES]. 